\title{
Caractériser les formes d'interaction entre filières agricoles et territoires : quelles méthodes ?
}

\author{
Sophie Madelrieux ${ }^{1, *}$, Nicolas Buclet ${ }^{2}$, Philippe Lescoat ${ }^{3}$ et Marc Moraine ${ }^{4}$ \\ ${ }^{1}$ Université Grenoble Alpes, IRSTEA, UR DTGR, 2 rue de la papeterie, BP76, 30402 St Martin d'Hères Cedex, France \\ ${ }^{2}$ Université Grenoble Alpes, IUG, 14 Avenue Marie Reynoard, 38100 Grenoble, France \\ 3 AgroParisTech, 16 rue Claude Bernard, 75231 Paris Cedex 05, France \\ ${ }^{4}$ ISARA-Lyon, 23 rue Jean Baldassini, 69364 Lyon cedex 07, France
}

\begin{abstract}
Résumé - Le processus de globalisation s'accompagne d'une importante restructuration des filières agricoles, se traduisant par une déconnexion de plus en plus forte entre opérateurs des filières et ceux des territoires, et entre lieux de production et usages des ressources locales. Les opérateurs des filières utilisent des ressources des territoires, mais n'y opèrent souvent qu'une partie de leur activité, et ne se soucient pas toujours de leur impact local, environnemental comme socioéconomique. Il y a un réel enjeu socioécologique à reconnecter les dynamiques des filières agricoles à celles des écosystèmes, à l'échelle des territoires, là où les activités de production prennent place. Pour cela, il est nécessaire de comprendre au préalable les formes d'interaction entre filières et territoires, pour pouvoir ensuite agir dans une perspective de développement durable. Nous avons élaboré un cadre d'analyse des formes d'interaction entre filières et territoires, interrogeant les formes d'ancrage des filières agricoles aux territoires, les formes de dépendance des territoires, pour leurs filières, vis-à-vis de ressources et opérateurs exogènes, ou d'autres territoires, enfin les formes d'empreinte des filières agricoles pour les territoires ou de la production agricole du territoire pour le reste du monde. Nous proposons ici une revue ciblée de la littérature sur les différentes méthodes existantes permettant de traiter ces différentes dimensions et leurs déclinaisons selon l'enjeu considéré : environnemental ou socioéconomique. Les approches biophysiques d'un côté et socioéconomiques de l'autre restent largement dissociées, ce qui nous amène à discuter de l'opérationnalité de ce tour d'horizon pour la communauté scientifique. Nous formulons également quelques recommandations, notamment pour éviter l'écueil du « localisme ».
\end{abstract}

Mots clés : filière / territoire / flux / secteur agricole / méthode

\begin{abstract}
Characterizing the forms of interaction between agricultural value chains and territories: what methods? Globalization comes along with an important restructuring of the agricultural value chains, resulting in an ever more important disconnection between operators of the supply chains and those of the territories, and between production sites and use of local resources. Operators of supply chains, which make use of local resources, often operate only a small part of their activity locally and do not always care about their environmental or socioeconomic impacts. There is a major challenge to reconnect supply chains' and ecosystems' dynamics at territory level. First, it requires understanding the types of interactions between supply chains and territories, to be able to design pathways for their sustainable co-evolution. We developed an analytical framework presenting forms of interactions between supply chains and territories, questioning the types of: anchoring of the agricultural activities in territories; dependence of territories to exogenous resources, operators or territories; footprints of the agricultural activities on local territory or on the rest of the world. We propose here a review of existing methods handling these different dimensions and their versions according to the considered issue: environmental or socioeconomic. Biophysical and socioeconomic approaches remain largely separated, what brings us to discuss the scientific gap to fill for reaching operational effectiveness of a potential integrative approach, while avoiding the pitfall of "localism".
\end{abstract}

Keywords: supply chain / territory / flow / agricultural sector / methods

\footnotetext{
*Auteur de correspondance : sophie.madelrieux@irstea.fr
} 


\section{Introduction}

\subsection{Les formes d'interaction entre filières agricoles et territoires en question}

Selon les pronostics démographiques et climatiques pour les prochaines décennies, le défi alimentaire mondial consistera à satisfaire une demande croissante par une production agricole durable (Rastoin et Ghersi, 2010). Ce défi prend particulièrement sens lorsqu'on observe l'évolution des activités d'élevage. La croissance de la demande en produits animaux dans les pays en développement (Agrimonde, 2009) s'inscrit dans une compétition internationale (ex. " usines à lait » en Chine ou aux États-Unis), et s'accompagne d'une croissance de l'utilisation de ressources peu renouvelables. Cela interroge l'utilisation des ressources et leur épuisement potentiel, les impacts sur les écosystèmes (perte de biodiversité, eutrophisation des milieux aquatiques), et les émissions de gaz à effet de serre (Steinfeld et al., 2006). Les activités d'élevage sont également questionnées par la surconsommation de viande dans les pays développés (Solagro, 2014), les modèles de production et le rapport aux animaux - mise en accusation de l'élevage industriel et des conditions d'abattage par le grand public - (Porcher, 2004 ; Harrison et al., 2013).

De façon plus générale, l'internationalisation des marchés et l'évolution de la consommation (demande de produits peu coûteux, standardisés et souvent transformés) ont eu une influence sur les pratiques agricoles : dynamiques d'agrandissement, spécialisation, intensification, délocalisation, recours à des capitaux extérieurs, spécialisations régionales des productions. Entre autres conséquences, on observe un pilotage de plus en plus fort des filières agroalimentaires par l'aval (opérateurs de la transformation et de la distribution), un découplage entre systèmes de production et usage des ressources locales, les systèmes étant de plus en plus artificialisés et basés sur le recours à des intrants. Ces transformations affectent les écosystèmes locaux, notamment par un accroissement des flux de matières et d'énergie et l'absence de bouclage des cycles des matières (Nesme et Withers, 2016). Elles induisent d'importantes dépenses énergétiques, et affectent la vie économique et sociale des territoires (Buclet, 2011).

Les filières utilisent des ressources (ex. : matières premières, force de travail) des territoires, mais n'y opèrent souvent qu'une partie de leur activité, et ne se soucient pas toujours de leur impact local. Bien entendu, les situations observables sont assez diverses. Il existe une pluralité de réponses des territoires aux dynamiques des filières : des territoires « subissent », d'autres construisent leur autonomie, tandis que d'autres encore font valoir des avantages comparatifs. Mais globalement, on observe que l'autonomie et la souveraineté des territoires se réduisent face aux marchés agricoles et aux entreprises de l'agro-industrie (Van der Ploeg, 2008). Certains acteurs de territoires (élus, chargés de développement territorial, associations), porteurs d'enjeux de développement socioéconomique et environnementaux, cherchent à retrouver une place dans la maîtrise de ce système (Brand et Bonnefoy, 2014). Il y a un réel enjeu socioécologique (Ostrom, 2009) à reconnecter les dynamiques des filières agricoles à celles des écosystèmes, à l'échelle des territoires, là où les activités de production prennent place. Pour cela, il est nécessaire de comprendre au préalable les formes d'interaction entre filières et territoires, pour pouvoir ensuite agir dans une perspective de développement durable.

\subsection{Vue d'ensemble des types d'analyse des formes d'interaction entre filières et territoires}

Dans un autre article de ce même numéro (Madelrieux et al., 2017), nous avons élaboré un cadre d'analyse des formes d'interaction entre filières et territoires, dans lequel nous distinguons :

- les formes d'ancrage des filières agricoles aux territoires ;

- les formes de dépendance des territoires, pour leurs filières (productions agricoles et valorisations), vis-à-vis de ressources et d'opérateurs exogènes, ou d'autres territoires ;

- les formes d'empreinte des filières agricoles sur les territoires ou de la production agricole du territoire sur le reste du monde.

L'analyse des formes d'ancrage permet de resituer la façon dont les filières s'appuient sur l'usage des ressources locales et les valorisent (propriétés des écosystèmes, ressources socioéconomiques ou culturelles) et sont reliées entre elles ou à d'autres activités localement (bouclages de flux, offre d'un bouquet de produits et services).

Saisir les formes de dépendance permet de compléter l'analyse des formes d'ancrage, par celles des intrants utilisés par les filières, et des centres de décision et stratégies des opérateurs échappant à la gouvernance locale. Il peut en effet se trouver des filières ancrées localement d'un point de vue socioéconomique mais qui le sont peu écologiquement, et qui peuvent être fortement dépendantes d'intrants venant d'ailleurs. On peut également trouver des productions très ancrées écologiquement parlant, mais dépendantes de filières pilotées à une échelle nationale et donc également fragilisées par de potentielles décisions prises en dehors de toute considération stratégique pour le territoire.

Mesurer les empreintes des formes d'interaction entre filières et territoires permet d'en proposer une évaluation environnementale et socioéconomique. Il peut s'agir de mesurer les ressources mises en jeu, les impacts, ou encore les services « rendus » ou richesses créées, soit par les filières agricoles pour les territoires, soit de la production agricole d'un territoire et de sa valorisation pour le reste du monde.

Caractériser les formes de dépendance, d'ancrage et d'empreinte renvoie à des questions et types d'analyses spécifiques selon l'enjeu considéré : environnemental ou socioéconomique (Tab. 1). Nous proposons ici une revue ciblée de la littérature sur les différentes méthodes existantes permettant de traiter ces différentes dimensions (sans rentrer dans les fondements et principes de chacune).

Dans ce qui suit, nous employons le terme «territoire » pour désigner un périmètre théorique de " concernement » d'acteurs liés au devenir des activités agricoles d'une zone géographique (la délimitation du périmètre d'étude est une question majeure que nous reprenons en discussion). Pour les filières agricoles, nous considérons principalement les exploitations agricoles et les maillons directement à leur amont et à leur aval dans la chaîne agroalimentaire. 
Tableau 1. Types d'analyse des formes d'interaction entre filières agricoles et territoires par rapport à des enjeux environnementaux et socioéconomiques.

Table 1. Types of analysis of the forms of interaction between agricultural sectors and territories with regard to environmental and socioeconomic issues.

\begin{tabular}{lll}
\hline & Enjeux environnementaux & Enjeux socioéconomiques \\
\hline Types de flux analysés & $\begin{array}{l}\text { Flux et stocks de matières, de substances } \\
\text { (ex. : N, P, K, C...), d'énergie, d'eau }\end{array}$ & Flux et stocks de ressources matérielles (intrants, \\
& & équipements production agricole brute, puis produits \\
& transformés...) et immatérielles (information, savoir- \\
faire...), de capitaux, de main-d'œuvre...
\end{tabular}

Ancrage

Question

Types d'analyses

Dépendance

Question

Types d'analyses

Empreinte

Question

Types d'analyses

\author{
Ancrage écologique \\ Analyse de l'usage des ressources locales et des \\ processus écologiques mobilisés (diversité et \\ complémentarités) \\ Analyse des liens aux autres activités ou fonctions \\ $\mathrm{du}$ territoire et des bouclages de flux (symbioses)
}

\begin{abstract}
Ancrage socioéconomique
Analyse de la gouvernance locale des filières agricoles Analyse des liens socioéconomiques aux autres activités économiques ou fonctions du territoire

Analyse des dépendances aux opérateurs extérieurs associés aux flux, des dépendances entre acteurs
\end{abstract}

Dépendance à des ressources et opérateurs extérieurs ou à d'autres territoires Analyse du poids des dépendances/autonomie par rapport à des flux entrants/sortants Analyse des dépendances : aires et distances d'approvisionnement

\section{Empreinte environnementale \\ Empreinte socioéconomique}

Évaluation des ressources (« naturelles »/socioéconomiques) mises en jeu dans la production agricole d'un territoire et sa valorisation

Mesure des impacts (environnementaux/socioéconomiques) : des filières agricoles pour le territoire ; de la production agricole d'un territoire pour le reste du monde

Évaluation des services environnementaux rendus Évaluation des types de « richesses » générées par les par les filières agricoles du territoire filières agricoles pour le territoire et synergies avec les autres activités

\section{Caractériser des formes d'interaction entre filières et territoires relativement à des enjeux environnementaux}

Il s'agit ici d'étudier les flux échangés avec l'environnement - intentionnellement (flux de matières) ou non (flux de substances, d'énergie...) - du fait du fonctionnement des filières.

\subsection{Formes d'ancrage}

L'analyse de l'usage des ressources et des flux permet de préciser la façon dont la production s'appuie sur les services des écosystèmes (par ex. : fertilité des sols, production des couverts végétaux). Balent et al. (2015) proposent une méthode d'analyse de la valorisation des services fourragers par les éleveurs pour réfléchir à une intensification « écologique » des modes de production. L'analyse des liens entre filières agricoles, notamment les liens entre cultures et élevages à l'échelle d'un territoire, ou avec les filières non agricoles, permet de réfléchir au bouclage des cycles de la matière localement, à la diversification des activités et leurs complémentarités. Moraine et al. (2016) proposent un cadre d'analyse des formes d'interaction entre culture et élevage à l'échelle de territoires. Simboli et al.
(2015) proposent une synthèse de travaux sur des symbioses dans des agri-food clusters et des liens mis en place localement entre activités, pour répondre à des problématiques énergétiques ou de gestion des déchets. Ils citent par exemple les travaux d'Abuyuan et al. (1999), où les résidus des activités agricoles sont utilisés pour générer des produits comme du biogaz, de la fibre, des fertilisants liquides riches en nutriments, qui sont eux-mêmes utilisés comme intrants localement dans des papeteries ou exploitations agricoles. Ces symbioses sont une façon d'ancrer localement des filières agricoles par rapport à des enjeux environnementaux (mais aussi socioéconomiques).

\subsection{Formes de dépendance}

Analyser les formes de dépendance des territoires, pour leurs filières agricoles, à des ressources extérieures au périmètre étudié peut passer par une évaluation du poids des dépendances à des flux entrants (Barles, 2014). Les approches existantes analysent les flux de substances (ex. : N, $\mathrm{P}, \mathrm{K}$ ) et d'énergie en lien à des problèmes environnementaux (émission de gaz à effet de serre, pollution des sols et de l'eau) et de finitude des ressources non renouvelables. Fernandez-Mena et al. (2016) proposent par exemple une revue des méthodes de modélisation et d'évaluation des flux de nutriments dans les filières agricoles. D'autres types 
d'analyses peuvent permettre de préciser la nature des dépendances, les aires et distances d'approvisionnement d'un territoire en énergie, en alimentation ou en eau (Billen et al., 2012). Cela permet de caractériser et mesurer l'interdépendance de territoires entre eux, de localiser les chaînes d'approvisionnement d'un territoire donné pour un flux donné, et de mettre en évidence des paradoxes (ex., ce qui est produit localement et quitte le territoire et ce qui entre pour le même usage).

\subsection{Formes d'empreinte}

Les calculs d'empreintes environnementales des filières agricoles pour les territoires peuvent porter sur :

- l'étendue des terres agricoles (empreinte spatiale, qui permet de mesurer l'emprise spatiale des exploitations agricoles dont le siège est situé dans le périmètre étudié) ;

- le volume des prélèvements d'eau et le taux moyen de mobilisation des apports d'origine pluviale (empreinte hydrique) ;

- le tonnage de substances comme l'azote (empreinte azotée brute), dont le partage entre la production d'aliments et les pertes environnementales dépend de la manière dont fonctionnent les agrosystèmes en présence (Chatzimpiros, 2011);

- les besoins en énergie pour obtenir un produit (Wilfart et al., 2012).

Parmi la diversité des méthodes d'évaluation d'impacts environnementaux (Van der Werf et al., 2011), nous retenons les méthodes d'analyse de cycle de vie (ACV), qui évaluent les impacts environnementaux d'un produit, d'un service ou d'un système tout au long de sa « vie » (Fuchs et al., 2011). Leur spatialisation permet de préciser des impacts pour différentes zones d'un territoire (Nitschelm et al., 2016). L'ACV territoriale (Loiseau, 2014), quant à elle, propose un cadre méthodologique qui permet une évaluation multicritère des systèmes de production ou filières agricoles d'un territoire, mais aussi des produits et services rendus par ces systèmes (ex. : taux de déchets traités, superficie en zone Natura 2000...).

L'analyse des services écosystémiques est une autre façon d'évaluer les services rendus par les filières agricoles. Par exemple Ryschawy et al. (2015) évaluent les services rendus par l'élevage à l'échelle nationale (ex. pour les aspects environnementaux : maintien des prairies - temporaires et permanentes - , qualité de l'eau...), et visent à les décliner localement. Dobremez et al. (2015), quant à eux, mesurent des services d'approvisionnement ou de régulation à l'échelle de parcelles, et visent à extrapoler à l'échelle territoriale.

\section{Caractériser des formes d'interaction entre filières et territoires relativement à des enjeux socioéconomiques}

Par rapport à des enjeux socioéconomiques, il s'agit d'étudier les flux intentionnellement échangés, en vue de la création de « richesses » (prises ici dans un sens large et non purement monétaire) : ressources matérielles (intrants, équipements, production agricole brute, puis produits transformés), immatérielles (information, savoir-faire...), capitaux ou main-d'œuvre. Mais il s'agit aussi d'analyser la façon dont ils sont structurés et inscrits dans des systèmes d'acteurs qui les pilotent et les régulent (Buclet, 2015).

\subsection{Formes d'ancrage}

L'analyse des formes d'ancrage socioéconomique vise à caractériser la façon dont la production et sa valorisation s'appuient sur l'usage de ressources territoriales (matérielles comme immatérielles) et sont reliées à d'autres activités locales, renforçant ainsi la richesse du territoire. Parmi les nombreux travaux sur l'ancrage territorial des filières ou des systèmes alimentaires, notamment des systèmes agroalimentaires localisés (Sanz Cañada et Muchnik, 2011), nous discernons trois types d'approches, selon les focales (stratégie d'entreprise, qualification de ressources, lien à la consommation) :

- l'analyse des stratégies d'entreprises par rapport au lieu (Saives, 2002) et aux concurrences spatiales dans un contexte de globalisation (Hannachi et al., 2010). Saives (2002) distingue différents types de comportements spatiaux des entreprises : les comportements de localisation, qui renvoient au choix d'un environnement pour la firme et reposent sur des critères d'accessibilité aux facteurs de production et/ou aux marchés de consommation, et les comportements de territorialisation qui consistent à développer des ressources en lien avec le territoire. Cela peut amener les entreprises à activer et spécifier des ressources territoriales et à mettre en place des coordinations situées (Colletis et Pecqueur, 2005) ;

- l'analyse de ces processus de construction de ressources territoriales à partir de la valorisation de produits agricoles, jusqu'à celle de paniers de biens et de services (Mollard et Pecqueur, 2007), dont celles des processus de qualification territoriale de produits alimentaires (Ilbery et al., 2005) ; - l'analyse des liens entre la production, ces processus de qualification, et la consommation (Sanz Cañada et Muchnik, 2011), et des proximités sociospatiales entre producteurs et consommateurs (Deverre et Lamine, 2010).

Baritaux et al. (2016) montrent que le concept d' « ancrage » (ou en anglais embeddedness) est largement mobilisé dans cette littérature. Il est emprunté à la sociologie économique, avec deux conceptions particulièrement mobilisées : l'ancrage social (Granovetter, 2000) et l'ancrage spatial (Penker, 2006). L'ancrage écologique est beaucoup moins traité. L'ancrage écologique ou ecological embeddedness est défini comme la façon dont « the actors involved in food production businesses address the ecological elements of food production and how these subsequently shape the process of exchange, from the perspective of both producers and consumers " (Morris et Kirwan, 2011). Ces travaux considèrent majoritairement des systèmes sous indication géographique ou alternatifs. Des travaux émergent pour tracer le local et sa signification dans des systèmes conventionnels (Forney et Häberli, 2014).

Les interactions entre filières agricoles et autres activités d'un territoire sont moins traitées, si ce n'est à travers les notions de paniers de biens et de services (Mollard et Pecqueur, 2007). Des propositions émergent en écologie territoriale pour saisir les interactions entre sous-systèmes créateurs de richesses au niveau d'un territoire, dont le système agroalimentaire (Buclet, 2015). 


\subsection{Formes de dépendance}

L'analyse des dépendances couvre les flux entrants, pour caractériser le poids d'un opérateur amont ou d'un territoire extérieur dans l'approvisionnement de la zone d'étude. Par exemple la dépendance d'exploitations d'élevage d'un territoire à des approvisionnements en fourrage (concentrés et paille) a été étudiée par Moraine et al. (2016). Elle peut aussi s'effectuer pour des flux sortants. Par exemple la fin des quotas laitiers (signant le passage d'une régulation publique et territorialisée des volumes et des prix, à une gestion privée par les entreprises de l'aval) s'accompagne d'un relâchement de l'ancrage de la production au territoire, d'une dépendance accrue aux cours volatils des marchés internationaux et aux opérateurs aval (Perrot et al., 2015). Pour répondre aux interrogations d'acteurs sur le devenir de leur production laitière dans ce contexte, Madelrieux et Lescoat (2016a) réalisent une analyse des flux de lait permettant de saisir le poids des opérateurs avals extérieurs dans la valorisation de la production du territoire, et montrent en quoi la gouvernance des filières agricoles échappe aux acteurs du territoire. Des méthodes d'analyse des systèmes d'acteurs, notamment de leurs stratégies, positions (Brullot et al., 2014) et de leurs proximités (Gilly et Lung, 2005) peut permettre d'aider à caractériser et qualifier ces relations entre acteurs.

\subsection{Formes d'empreinte}

En ce qui concerne les ressources socioéconomiques mises en jeu dans la production agricole d'un territoire et sa valorisation, nous pouvons citer les travaux de Lang et al. (2015) qui évaluent les emplois liés à l'élevage. Cette méthode a été mise au point pour calculer les emplois liés à l'élevage français, selon les acteurs des filières (les élevages, les fournisseurs des élevages, les industries aval de transformation, la distribution, la logistique, les services publics et parapublics), et selon les filières (lait : bovin, ovin, caprin; viande ruminants : bovin, ovin viande, veau de boucherie; granivore : porc, volaille chair et lapins, volaille œuf). Cette méthode appliquée à l'échelle nationale peut se décliner sur des périmètres plus restreints pour évaluer l'emploi dans un territoire lié aux filières d'élevage.

Paus et Reviron (2010) proposent une revue des méthodes visant à mesurer l'impact territorial de filières agroalimentaires « locales ». Elles distinguent les méthodes dites « objectives » (indicateurs d'impacts), d'autres dites « subjectives » (effets perçus). Elles montrent que de nombreuses études traitent principalement de la performance économique via le calcul de différentiels de revenus, d'emplois et d'investissement. Certaines seulement se sont attachées à mesurer les impacts environnementaux, paysagers, ou encore la dynamique sociale. La mesure des impacts socioéconomiques concerne également ceux de la production agricole d'un territoire pour le reste du monde, avec les risques d'appauvrissement de certaines zones du fait de jeux de concurrence entre territoires pour une même production (Dufumier et Hugon, 2008), ou les opportunités d'enrichissement en fournissant une production à haute valeur ajoutée, mais qui ne bénéficie pas aux producteurs ou au territoire de production. C'est ce qui est notamment en jeu dans la mise en place du " commerce équitable», par exemple pour la café (Daviron et Ponte, 2007). Les méthodes d'ACV sociale se développent (Macombe, 2013) pour prévoir les effets sociaux d'un changement dans une filière à un endroit donné sur le reste de la filière et les zones concernées, en particulier quand elles concernent des filières internationales de produits agricoles.

Concernant l'évaluation des services et "richesses » générées par les filières agricoles pour le territoire, on peut citer les méthodes d'ACV territoriale (Loiseau, 2014) qui permettent également de mesurer des services d'ordre socioéconomique (ex. : produit brut du secteur agricole, fourniture d'emplois, taux de qualification...). Dans la lignée des travaux sur les ressources territoriales, on trouve aussi des travaux sur l'évaluation de la rente territoriale générée (Hirczak et al., 2008).

\section{Discussion}

\subsection{Opérationnalité de ce tour d'horizon pour la communauté scientifique}

\subsubsection{Vers une analyse systémique des relations entre filières et territoires}

À travers notre cadre d'analyse, nous avons proposé des références méthodologiques permettant de traiter différentes dimensions des interactions entre filières agricoles et territoires (formes d'ancrage, de dépendance, et d'empreinte) en lien à des enjeux environnementaux et socioéconomiques. La linéarité du propos et le rendu du cloisonnement des approches dans le Tableau 1 ne sont pas qu'un effet de la présentation. Les approches biophysiques d'un côté, et socioéconomiques de l'autre, restent en effet largement dissociées (Buclet, 2015).

Un enjeu est donc de parvenir à articuler les concepts et les méthodes présentés. Les difficultés sont celles immanentes à tout travail mettant en jeu différentes disciplines, chaque approche abordant, dans son paradigme (avec ses concepts et postures, points de vue, langages, connaissances, contraintes techniques de collecte des données, méthodes quantitatives ou qualitatives) les interactions entre filières et territoires de façon différente. C'est l'enjeu de l'interdisciplinarité de construire « une démarche d'assemblage dialogique des apports disciplinaires nécessaires à l'analyse d'un objet complexe » (Jollivet, 2002). C'est en explicitant où se situent les « charnières » entre les approches que nous serons à même d'identifier les zones d'irréductibilité propres aux disciplines et ce qu'il faudrait au contraire travailler pour accroître la dimension dialogique de la démarche générale.

Cela nous semble possible, dans le cadre théorique des systèmes socioécologiques (McGinnis et Ostrom, 2014). Partant de ce cadre général, nous pouvons donner des pistes de réflexion sur ce qui pourrait faire charnière. Appréhender le " métabolisme » des interactions entre filières et territoires, notamment les flux et stocks (Erb, 2012 ; Buclet, 2015), peut permettre de faire le lien et de comprendre comment ces flux et stocks répondent à la fois au fonctionnement des écosystèmes et à celui des systèmes sociotechniques. Cela peut notamment permettre de comprendre les interactions entre :

- l'utilisation des ressources et les écosystèmes (et par làmême les empreintes environnementales) ;

- l'utilisation des ressources et les systèmes d'acteurs qui les valorisent (et par là-même les empreintes socioéconomiques).

Les évolutions du métabolisme permettent, quant à elles, de comprendre en dynamique les interactions entre les 
évolutions des écosystèmes d'une part et les mutations sociotechniques d'autre part.

Certains outils existent mais doivent pouvoir être renforcés et testés dans des situations d'analyse et de prospective territoriale. Par exemple une cartographie de flux pourrait permettre d'identifier leurs circulations, les activités et acteurs associés. Sur cette base pourrait se réaliser le diagnostic des formes d'ancrage, de dépendance, et d'empreinte. Pour ces dernières, il s'agirait de convertir les flux de matière en multiples catégories de pression environnementale (Courtonne, 2016) ou de suivre des flux monétaires (Ruault, 2014) adossés à ces flux de matières (un flux monétaire s'adosse à un flux réel, à l'inverse tous les flux d'énergie et de matière n'ont pas une valeur d'échange effective), pour évaluer la richesse créée.

Cela ouvre sur un programme de recherche, à l'échelle des territoires, en agronomie et écologie territoriale.

\subsubsection{Fédérer une communauté de chercheurs}

Mettre en œuvre ce programme de recherche nécessite de fédérer une communauté de chercheurs sur ces questions, autour d'une posture interdisciplinaire et d'un pluralisme conceptuel (Costanza, 1989). L'enjeu est que cela puisse permettre de contribuer à la compréhension de l'objet de la recherche, bien sûr, mais aussi de faire progresser méthodologiquement la façon d'assembler, voire d'intégrer, les apports disciplinaires (Jollivet et Legay, 2005), afin de dépasser une méthode cumulative de concepts, d'indicateurs, d'outils, et de fournir une démarche d'analyse opérationnelle des interactions entre filières et territoires. Le cadre d'analyse proposé, bien que sommaire, permet de situer les différentes approches et communautés associées, et de situer leurs articulations possibles. La façon dont chacun peut apporter sa contribution à l'analyse des interactions entre filières et territoires peut-elle faciliter un tel rapprochement entre chercheurs pour une analyse plus systémique et intégratrice des interactions entre filières et territoires ? Sur cette base, les contributions des différents courants de recherche pourraient-elles permettre :

- des façons complémentaires d'éclairer l'objet qui s'enrichissent mutuellement pour en construire une connaissance originale ;

- l'enrichissement du cadre d'analyse et d'intégration dans une co-construction au sein de cette communauté ?

\subsection{Recommandations}

\subsubsection{Délimitation du périmètre d'étude}

Analyser les interactions entre filières et territoires met en évidence la multiplicité et l'enchevêtrement des périmètres (enjeux, systèmes d'acteurs et de gouvernance des filières et des territoires). Le premier défi de cette analyse est donc de délimiter le périmètre d'étude pertinent pour comprendre et agir (Buclet, 2015). La notion de territoire, polysémique, relève d'un construit social et ses limites peuvent difficilement être établies a priori (Laganier et al., 2002), que ce soit sur des bases administratives, géologiques, hydrologiques ou encore climatiques. Il s'agit de bien distinguer le périmètre d'étude avec une délimitation géographique permettant de mesurer des flux qui entrent et sortent, des territoires qui se construisent par les réseaux et représentations sociales. La délimitation de départ, si elle permet de préciser les ancrages, dépendances, empreintes, ne réduit pas la zone à un système clos. Comme le dit Wassenaar (2015) : " isolating a segment of reality for analysis is not equal to an isolated analysis of that segment $»$. Cela permet au contraire d'analyser la place des activités agricoles du périmètre étudié dans des réseaux plus larges, écologiques et socioéconomiques, à différentes échelles. Le périmètre d'étude peut être défini, par les chercheurs, par rapport à une question de recherche (devenir de la filière laitière en zones de montagne), ou à partir du périmètre de concernement d'acteurs d'un territoire (ex. : demande d'un parc naturel régional), ou en interaction dans le cadre d'une recherche en partenariat (devenir de la filière laitière dans le périmètre montagneux du parc naturel régional de Chartreuse ; Madelrieux et Lescoat, 2016b).

\subsection{2 Éviter l'écueil du « localisme »}

Comprendre les formes d'interaction entre filières et territoires est particulièrement important pour les filières longues et conventionnelles dont la territorialisation est peu abordée dans les travaux, par rapport aux filières sous indication géographique ou « alternatives ». Pour autant, il ne faut pas tomber dans le "local trap 》 (Forney et Häberli, 2016 ) et produire des raccourcis entre local et durable. Forney et Häberli (2016); analysent comment se construit le « local », comment il est négocié dans des initiatives impliquant des producteurs, et est utilisé comme moyen de reconnecter au lieu. Il ressort trois formes d'ancrage socioéconomique et d'expression du local : le local comme « provenance » (c'est la provenance du produit qui le qualifie, mais aucunement ses caractéristiques, et ce peut être des produits standard) ; comme « origine » (c'est la spécificité du lieu, notamment par un rattachement à une tradition identitaire, qui qualifie le produit) ; comme « proximité et solidarité » (c'est la mise en réseau de producteurs, leur proximité et solidarité, et le lien plus direct au consommateur, qui sont mis en avant). Cela renvoie à différentes stratégies guidant les initiatives : stimuler l'économie locale, renforcer l'autonomie par rapport à l'industrie et les liens entre producteurs, construire une identité dans un contexte de compétition. Les auteurs explorent également comment ces stratégies peuvent ouvrir sur des transformations plus profondes. Différents travaux sur les systèmes agroalimentaires localisés identifient la propension à un " localisme défensif » qui renforce les frontières sociales et identitaires, en accordant bien peu d'attention à l'environnement et la justice sociale. Le cadre d'analyse proposé vise justement à fournir des clés pour éviter ces écueils, en élargissant le champ de l'analyse de la construction du « local » à partir de l'usage des écosystèmes locaux, et des dimensions complémentaires sur les formes de dépendance et d'empreinte. Il peut, en effet, se trouver des formes d'interaction ancrées localement d'un point de vue socioéconomique, mais qui le sont peu écologiquement, et qui peuvent être aussi fortement dépendantes d'intrants venant d'ailleurs, ou avec un impact environnemental non négligeable. Cette analyse, complexe mais nécessaire, peut permettre d'informer de manière robuste les relations entre « local» et « durable ». 


\section{Conclusion et perspectives}

L'économie des filières renvoie à une économie de flux de natures variées (cultures, élevages, co-produits...), fait appel à des procédés et opérateurs multiples, et s'adresse à des marchés divers (alimentation, matériaux, chimie, énergie...), eux-mêmes inscrits dans une diversité d'échelles géographiques. Si les ressources ne sont pas également réparties dans l'espace, tous les espaces ont potentiellement des ressources à condition de les faire émerger et de les valoriser (Gumuchian et Pecqueur, 2007). Ces dynamiques d'activation et/ou de spécification de ressources peuvent faire l'objet d'une captation industrielle de produits ancrés au terroir, ou s'appuyer sur des logiques locales mais adossées à des produits et procédés génériques, ou encore sur la mise en place de filières courtes territorialisées. D'autres dynamiques purement " aterritoriales » existent, elles aussi adossées à des produits et procédés génériques, mais ne permettent pas l'activation de telles ressources. La revue de littérature présentée ici montre l'intérêt de se doter d'une grille de lecture des interactions entre filières et territoires, de méthodes et outils opérationnels, pour analyser la nature et l'intensité des liens entre filières et territoires, et déterminer les dimensions de la territorialisation des filières, les bénéfices associés, les limites potentielles, et les perspectives de durabilité.

L'analyse des jeux d'acteurs permet de mieux comprendre également en quoi les territoires et les filières peuvent être des leviers ou des obstacles à la territorialisation de la production ou à sa transition agroécologique (Fares et al., 2012). Au-delà de l'analyse, ces outils peuvent permettre de développer des modes de coordination entre acteurs des filières et des territoires, et de gouvernance favorisant la gestion durable des ressources d'un territoire (Ostrom, 1990), à travers des dispositifs de recherche participative (Gouttenoire et al., 2014), favorisant les interactions entre chercheurs et acteurs (Hubert et al., 2004). Clarifier les notions et proposer un cadre de questionnement et d'analyse est, pour nous, un premier pas pour formaliser des démarches plus opérationnelles. Le cadre d'analyse ébauché laisse pressentir une grande diversité des formes d'interaction, en tenant compte des interactions socioéconomiques mais aussi écologiques (ex. : parasitisme : les filières utilisent des ressources des territoires mais sans se préoccuper des retombées pour le territoire ; mutualisme : association positive et obligatoire pour les deux ; commensalisme : association positive pour l'un et indifférente pour l'autre ; compétition pour l'accès aux ressources... [Buclet et al., 2015]), qui restent largement à caractériser et mettre à l'épreuve de différents cas d'étude.

\section{Références}

Abuyuan A, Hawken I, Newkirk M, Willians R. 1999. Waste equal food: developing a sustainable agriculture cluster for a proposed resource recovery park in Puerto Rico. Yale F \& ES Bulletin 106: 303-349.

Agrimonde. 2009. Agricultures et alimentation du monde en 2050 : scénarios et défis pour un développement durable. Note de synthèse. Paris : Inra et Cirad. Disponible sur http://www.cirad.fr/ cirad_fr/publications-ressources/edition/etudes-et-documents/agri monde.
Balent G, Bertoni G, Boisdon I, Capitaine M, Colace MP, Dobremez L, et al. 2015. Quelles perspectives d'intensification écologique des services fourragers rendus par des prairies de moyenne montagne française ? Une analyse comparative. Fourrages 221: 3-14.

Baritaux V, Houdart M, Boutonnet JP, Chazoule C, Corniaux C, Fleury P, et al. 2016. Ecological embeddedness in animal food systems (re-)localisation: a comparative analysis of initiatives in France, Morocco and Senegal. Journal of Rural Studies 43: 13-26.

Barles S. 2014. L'écologie territoriale et les enjeux de la dématérialisation des sociétés : l'apport de l'analyse des flux de matières. Développement Durable et Territoires 5(1). DOI:10.4000/developpementdurable.10090.

Billen G, Barles S, Chatzimpiros P, Garnier J. 2012. Grain, meat and vegetables to feed Paris: where did and do they come from? Localising Paris food supply areas from the eighteenth to the twenty-first century. Reg Environ Change 12: 325-335.

Brand C, Bonnefoy S. 2014. Régulation politique et territorialisation $\mathrm{du}$ fait alimentaire : de l'agriculture à l'agri-alimentaire. Géocarrefour 89: 95-103.

Brullot S, Maillefert M, Joubert J. 2014. Stratégies d'acteurs et gouvernance des démarches d'écologie industrielle et territoriale. Développement Durable et Territoires 5 (1). Disponible sur http://developpementdurable.revues.org/10082.

Buclet N. 2011. Écologie industrielle et territoriale. Stratégies locales pour un développement durable. Villeneuve d'Ascq : Presses universitaires du Septentrion.

Buclet N, ed. 2015. Essai d'écologie territoriale. L'exemple d'Aussois en Savoie. Paris : CNRS Éditions.

Buclet N, Cerceau J, Debuisson M, Maillefert M, Métereau R, Pech P, et al. 2015. Création de richesses et réponses aux besoins de la population d'Aussois. In : Buclet N, ed. Essai d'écologie territoriale. L'exemple d'Aussois en Savoie. Paris (France) : CNRS Éditions, pp. 105-160.

Chatzimpiros P. 2011. Les empreintes environnementales de l'approvisionnement alimentaire : Paris et ses viandes et lait, $\mathrm{xix}^{\mathrm{e}}-\mathrm{xxi}^{\mathrm{e}}$ siècle. Thèse de doctorat, université Paris-Est. Disponible sur https://halshs.archives-ouvertes.fr/pastel-00834837/document.

Colletis G, Pecqueur B. 2005. Révélation de ressources spécifiques et coordination située. Revue Économie et Institutions 6-7: 51-74.

Costanza R. 1989. What is ecological economics? Ecological Economics 1: 1-7.

Courtonne JY. 2016. Évaluation environnementale de territoires à travers l'analyse de filières. Thèse de doctorat, université Grenoble-Alpes. Disponible sur https://tel.archives-ouvertes.fr/tel01421664/document.

Daviron B, Ponte S. 2007. Le paradoxe du café. Versailles : Éditions Quae.

Deverrs C, Lamine C. 2010. Les systèmes agroalimentaires alternatifs : une revue de travaux anglophones en sciences sociales. Économie Rurale 317: 57-73.

Dobremez L, Chazoule C, Loucougaray G, Pauthenet Y, Nettier B, Lavorel S, et al. 2015. Débats et controverses sur l'intensification fourragère dans le Vercors : quelles pratiques, quelles conceptions en jeu ? Fourrages 221: 33-45.

Dufumier M, Hugon P. 2008. Piques et polémiques les « émeutes de la faim » : du sous-investissement agricole à la crise socio-politique. Revue Tiers Monde 4 (196): 927-934.

Erb KH. 2012. How a socio-ecological metabolism approach can help to advance our understanding of changes in land-use intensity. Ecological Economics 76: 8-14.

Fares M, Magrini MB, Triboulet P. 2012. Transition agro-écologique, innovation et effets de verrouillage : le rôle de la structure organisationnelle des filières. Le cas de la filière blé dur française. Cahiers Agricultures 21: 34-45. 
Fernandez-Mena H, Nesme T, Pellerin S 2016. Towards an agroindustrial ecology: a review of nutrient flow modeling and assessment tools in agro-food systems at the local scale. Science of the Total Environment 543: 467-479.

Forney J, Häberli I. 2016. Introducing 'seeds of change' into the food system? Localisation strategies in the Swiss dairy industry. Sociologia Ruralis 56 (2): 135-156.

Fuchs F, Sidot G, Waroquiers C. 2011. Propositions pour faire évoluer les outils d'analyse de cycle de vie des produits d'origine agricole. Innovations Agronomiques 17: 213-226.

Gilly JP, Lung Y. 2005. Proximités, secteurs et territoires. Cahiers $d u$ GRES 9. 21 p. Disponible sur http://cahiersdugres.u-bordeaux4.fr/ 2005/ 2005-09.pdf.

Gouttenoire L, Taverne M, Cournut S, Hostiou N, Houdart M, Lardon S. 2014. Faciliter les échanges entre chercheurs sur les projets de recherche participative : proposition d'une grille d'analyse. Cahiers Agricultures 23: 205-212.

Granovetter M. 2000. Le marché autrement. Paris : Desclée de Brouwer.

Gumuchian H, Pecqueur B. 2007. La ressource territoriale. Paris : Éditions Economica.

Hannachi M, Coléno FC, Assens C. 2010. La collaboration entre concurrents pour gérer le bien commun : le cas des entreprises de collecte et de stockage de céréales d'Alsace. Revue Gérer et Comprendre 101: 16-25.

Harrison R, Carson R, Dawkins MS, Stamp Dawkins M, Webster J, Rollin BE, et al. 2013. Animal machines. London: Vincent Stuart Publishers.

Hirczak M, Moalla M, Mollard A, Pecqueur B, Rambonilaza M, Vollet D. 2008. From the model of basket of goods to a more general model of territorialized complex goods: concepts, analysis grid and questions. Canadian Journal of Regional Science 31 (2): 241-259.

Hubert B, Moulin CH, Roche B, Pluvinage J, Deffontaines JP. 2004. Quels dispositifs pour conduire des recherches en partenariat ? L'intervention d'une équipe de recherche au Pays basque intérieur. Économie Rurale 279: 33-52.

Ilbery B, Morris C, Buller H, Maye D, Kneafsey M. 2005. Product, process and place: an examination of food marketing and labelling schemes in Europe and North America. European Urban and Regional Studies 12 (2): 116-132.

Jollivet M. 2002. Relier les connaissances, transversalité, interdisciplinarité. Nature Sciences et Sociétés 10 (1): 78-95.

Jollivet M, Legay J.M. 2005. Canevas pour une réflexion sur une interdisciplinarité en sciences de la nature et sciences sociales. Nature Sciences et Sociétés 13: 184-188.

Laganier R, Villalba B, Zuindeau B. 2002. Le développement durable face au territoire : éléments pour une recherche pluridisciplinaire. Développement Durable et Territoires. 19 p. DOI:10.4000/ developpementdurable.774.

Lang A, Perrot C, Dupraz P, Tregaro Y, Rosner PM 2015. Les emplois liés à l'élevage français. Paris : Gis Élevage Demain. Disponible sur https://www.gis-elevages-demain.org/Media/fichiers/Rapportfinal-Emplois-lies-a-l-elevage.

Loiseau E. 2014. Élaboration d'une démarche d'évaluation environnementale d'un territoire basée sur le cadre méthodologique de l'analyse du cycle de vie (ACV). Application au territoire du bassin de Thau. Thèse de doctorat, Centre international d'études supérieures en sciences agronomiques de Montpellier. Disponible sur http://www.supagro.fr/theses/extranet/14-0008_Loiseau.pdf.

Macombe C, ed. 2013. ACV sociales. Effets socio-économiques des chaînes de valeurs. Montpellier : FRuiTROP.

Madelrieux S, Lescoat P. 2016a. Effects on territories of ending milk quotas. Exploratory findings from two contrasting case studies: the Niort Plain and the Chartreuse Massif. In: 12th European IFSA Symposium, 12-15 July 2016, Harper Adams University, UK.
Madelrieux S, Lescoat P. 2016b. Devenirs de la production laitière dans les territoires. Cas du parc naturel régional de Chartreuse. In : $3^{e}$ colloque international du CIST - En quête de territoire(s)? Looking for territories?, 17-18 mars 2016, Cité des territoires, Grenoble, France.

Madelrieux S, Buclet N, Lescoat P, Moraine M. 2017. Écologie et économie des interactions entre filières et territoires : quels concepts et cadre d'analyse ? Cahiers Agricultures 26: 24001.

McGinnis MD, Ostrom E. 2014. Social-ecological system framework: initial changes and continuing challenges. Ecology and Society 19 (2). 12 p. http://doi.org/10.5751/ES- 06387-190230.

Mollard A, Pecqueur B. 2007. De l'hypothèse au modèle du panier de biens et de services. Histoire succincte d'une recherche. Économie Rurale 300: 110-114.

Moraine M, Duru M, Therond O. 2016. A social-ecological framework for analyzing and designing integrated croplivestock systems from farm to territory levels. Renewable Agriculture and Food Systems 32 (1): 43-56. DOI:10.1017/ S1742170515000526.

Morris C, Kirwan J. 2011. Ecological embeddedness: an interrogation and refinement of the concept within the context of alternative food networks in the UK. Journal of Rural Studies 27 (3): 322-330.

Nesme T, Withers P. 2016. Sustainable strategies towards a phosphorus circular Economy. Nutr Cycl Agroecosyst 4: 259-264.

Nitschelm L, Aubin J, Corson MS, Viaud V, Walter C. 2016. Spatial differentiation in life cycle assessment (LCA) applied to an agricultural territory: current practices and method development. Journal of Cleaner Production 112 (4): 2472-2484.

Ostrom, E. 1990. Governing the commons: the evolution of institutions for collective action. Cambridge: Cambridge University Press.

Ostrom, E. 2009. A general framework for analyzing sustainability of social-ecological systems. Science 325 (5939): 419-422.

Paus M, Reviron S. 2010. Mesure de l'impact territorial d'initiatives agroalimentaires. Enseignement de deux cas suisses. Économie Rurale 315: 28-45.

Penker M. 2006. Mapping and measuring the ecological embeddedness of food supply chains. Geoforum 37 (3): 368-379.

Perrot C, Caillaud D, Chatellier V, Ennifar M, You G. 2015. La diversité des exploitations et des territoires laitiers face à la fin des quotas. Fourrages 221: 57-68.

Porcher J. 2004. Bien-être animal et travail en élevage. Paris : Educagri Éditions.

Rastoin JL, Ghersi G. 2010. Le système alimentaire mondial. Concepts et méthodes, analyses et dynamiques. Versailles : Éditions Quae.

Ruault JF. 2014. Mesure de la consommation de passage dans une approche circulaire de l'économie. In : L'effet de la consommation de passage sur le développement et l'intégration métropolitaine des territoires en Ile-de-France. Thèse de doctorat, université Paris-Est, p. 163-198. Disponible sur https://halshs.archivesouvertes.fr/tel-01149904.

Ryschawy J, Tichit M, Bertrand S, Allaire G, Plantureux S, Aznar O, et al. 2015. Comment évaluer les services rendus par l'élevage? Une première approche méthodologique sur le cas de la France. Inra Prod Anim 28 (1): 23-38.

Saives AL. 2002. Territoire et compétitivité de l'entreprise. Paris : L'Harmattan.

Sanz Cañada J, Muchnik J. 2011. Introduction : ancrage et identité territoriale des systèmes agroalimentaires localisés. Économie Rurale 2 (322): 4-10. 
Simboli A, Taddeo R, Morgante A. 2015. The potential of industrial ecology in agri-food clusters (AFCs): a case study based on valorisation of auxiliary materials. Ecological Economics 111: 65-75.

Solagro. 2014. Afterres 2050 : un scénario soutenable pour l'agriculture et l'utilisation des terres en France à l'horizon 2050. Disponible sur http://www.alimenterre.org/ressource/afterres2050-scenario-soute nable-lagriculture-et-lutilisation-terres-france.

Steinfeld H, Gerber P, Wassenaar T, Castel V, Rosales M, de Haan C. 2006. Livestock's long shadow. Environmental issues and options. Rome: FAO.
Van der Ploeg JD. 2008. Struggles for autonomy and sustainability in an era of empire and globalization. London: Earthscan.

Van der Werf HMG, Kanyarushoki C, Corson MS. 2011. L'analyse de cycle de vie : un nouveau regard sur les systèmes de production. Agricole. Innovations Agronomiques 12: 121-133

2015. Wassenaar T. Reconsidering industrial metabolism. From analogy to denoting actuality. Journal of Industrial Ecology 19 (5): 715-727.

Wilfart A, Corson MS, Aubin J, 2012. La méthode EMERGY : principes et application en analyse environnementale des systèmes agricoles et de production animale. Inra Prod Anim 25: 57-66.

Citation de l'article : Madelrieux S, Buclet N, Lescoat P, Moraine M. 2017. Caractériser les formes d'interaction entre filières agricoles et territoires : quelles méthodes ? Cah. Agric. 26: 24002. 